\title{
DISEÑO DE ESTRATEGIA DE CONTROL AVANZADO PARA SISTEMA DE CELDAS DE FLOTACIÓN EN EL TRATAMIENTO DE AGUAS DE PRODUCCIÓN DE PETRÓLEO Y GAS
}

\section{ADVANCED CONTROL STRATEGY DESIGN FOR FLOTATION CELLS SYSTEMS IN OIL AND GAS WATER PRODUCTION TREATMENT}

\author{
MSc. Javier Enrique Castellanos*, PhD. Rocco Tarantino Alvarado.** \\ MSc. Sandra Aranguren Zambrano.** \\ * Pacific Rubiales Energy. \\ Calle 110, No. 9-25, Torre Empresarial Pacific. Bogotá D.C. Colombia. \\ E-mail: jcastellanos@ pacificrubiales.com.co \\ ** Universidad de Pamplona, Ciudadela Universitaria. Pamplona. \\ Tel.: (+577) 568 5303, Fax: (+577) 568 5303, Ext. 156. \\ E-mail: \{rocco.tarantino, sandraranguren\}@unipamplona.edu.co
}

\begin{abstract}
Resumen: Una de las tareas más complejas en los separadores de flotación de aire inducido es el control de nivel. En este trabajo se desarrolla una estrategia de control avanzada aplicada a celdas de flotación en las instalaciones de petróleo y gas que requieren mejoras en el rendimiento de los lazos de control. Esta estrategia emplea sistemas de controlador PID adaptativo difusos y los ajustes de consigna se realiza a través de un modelo hidráulico. Se utiliza para la implementación el conjunto de controladores y software de Allen Bradley en un entorno industrial.
\end{abstract}

Palabras Clave: Separadores por flotación y aire, emulación de planta, estrategias de control avanzado.

\begin{abstract}
One of the most complex tasks in induced air flotation separators is the level control. In this work is developed an advanced control strategy applied for flotation cells in oil and gas facilities which requires improvement in control loops performance. This strategy employs fuzzy adaptive PID controller systems and set point adjustments are made through a hydraulic model. Is used for implementation the suite of drivers and software from Allen Bradley in industrial environment.
\end{abstract}

Keywords: Air flotation separators, plant emulation, advanced control strategies.

\section{INTRODUCCIÓN}

La flotación es uno de los métodos de separación de mayor uso en la industria minera y petrolera. Este proceso fue introducido en el año $1900 \mathrm{y}$ desde entonces se han llevado a cabo numerosos estudios de investigación y desarrollo de esta tecnología. Sin embargo, el proceso de flotación en la industria petrolera sigue siendo relativamente ineficiente en la medida en que la recuperación de hidrocarburos debe efectuarse en más de un ciclo de flotación. Las mayores ganancias económicas generadas por este proceso se deben a la implantación de estrategias de optimización (B.J. Shean, J.J. Cilliers et al. 2011). El mayor fluido producido en la extracción de petróleo y gas durante la vida de casi todos los pozos y yacimientos es el agua. Para la eliminación final de trazas de aceite en aguas de proceso, en algunos campos productores se emplean sistemas de flotación-filtración con celdas, los cuales deben contar con la capacidad de eliminación de aceite para disponer aguas de buena calidad para el ecosistema. 
El agua de producción entra y circula por mecanismos aireadores (figura1), ubicados al interior la de celda, cada mecanismo se encuentra en un compartimiento independiente donde se le ofrece al fluido un tiempo de residencia.

En la operación, cada mecanismo arrastra el aire dentro del agua de producción y circula el agua rica en aire a través de toda la celda, el aceite libre y sólidos suspendidos presentes en el agua de producción se adhieren a las burbujas de aire y son llevados a la superficie creando una disminución de la capa espumosa espesa que es removida por skimers o paletas dirigiéndolas hacia cajetillas colectoras, allí el crudo removido al proceso como se muestra en figura 1.

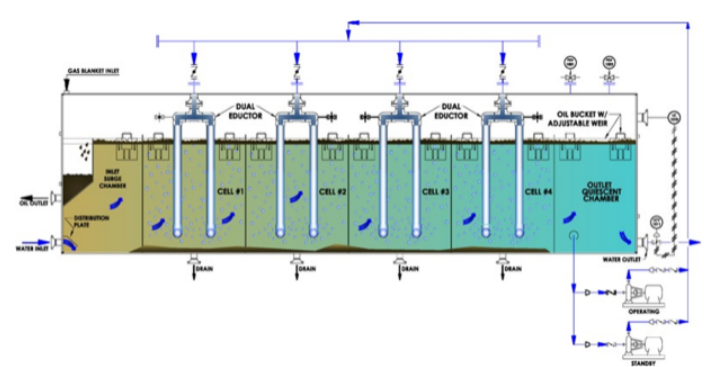

Fig. 1. Celda de flotación con arrastre de aire.

Debido a la complejidad del proceso de flotación estudiado, propia de su dinámica y de las perturbaciones asociadas, y dada la simplicidad de la estrategia de control PID establecida en el sistema se presenta inestabilidad de las variables de proceso, las cuales se reflejan en la deficiente remoción de aceite flotante y en el re-proceso de aguas.

Las falencias de remoción de aceite generan aumento de costos y tiempos operacionales para evitar impactos ambientales sobre las aguas de disposición final del campo de producción petrolera. Teniendo en cuenta estos antecedentes, se ha diseñado una estrategia de control, la cual combina técnicas de control adaptativas basadas en el criterio experto y adicionalmente ajuste de consignas de proceso basado en modelo dinámico de parte del sistema hidráulico.

\section{PROBLEMA DE CONTROL DEL SISTEMAS DE FLOTACIÓN-FILTRACIÓN}

Los circuitos de flotación en donde las interacciones son fuertes, los controladores PID no alcanzan requerimientos de alto desempeño; sin embargo se ha dispuesto en los últimos años una cantidad considerable de recursos, para desarrollar técnicas de control mejoradas para procesos basados en flotación (Jamsa-Journela et al., 2001)

El sistema de control de la planta se constituye por un simple lazo de control convencional. Debido a las características del proceso de flotación y las perturbaciones causadas por las corrientes de flujo aguas arriba y aguas abajo, se ve afectada la eficiencia del control de dicho proceso, generando alto oleaje de fluido, lo cual activa de manera intermitente las protecciones por alto nivel. Este comportamiento variable del nivel causa los siguientes problemas:

- Disminución de ciclo de vida de la válvula de control por continuo proceso de cierre $y$ apertura.

- Desnate inadecuado que genera mayor devolución de agua para reproceso, lo cual disminuye la capacidad nominal instalada del las facilidades de producción de petróleo.

- Desgaste de bombas hidráulicas de reproceso de crudo por alto contenido de agua, trabajando a propiedades de fluido fuera de diseño.

- Afectación ambiental negativa de aguas de disposición.

A pesar de varios avances en los controles de nivel desde la década de 1970, son escasos los reportes de sistemas de control de flotación avanzados, optimizados y en operación remota total (B. J. Shean, J. J. Cilliers et al., 2011).

En la figura 2, se presenta la tendencia de la variable de proceso nivel de la celda de flotación en un periodo de tiempo de cinco horas. En dicha tendencia se aprecia de manera clara la dificultad del sistema de control para seguir el set point; adicionalmente, se observan desviaciones del nivel por arriba y abajo, lo cual sugiere que la remoción de hidrocarburos se ha efectuado de manera irregular.

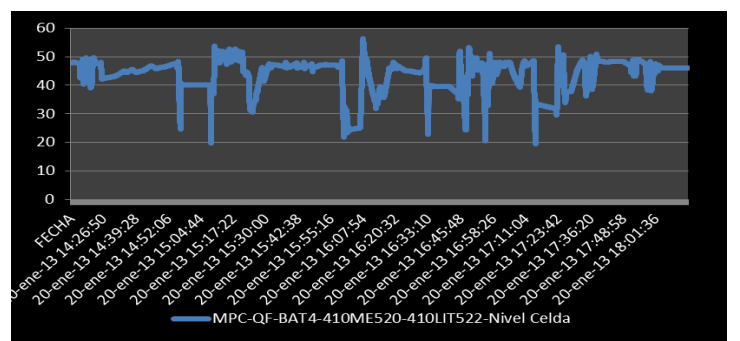

Fig. 2. Comportamiento perturbado de la variable de proceso nivel del sistema de flotación. 
En la figura 3 se presenta el comportamiento que asume la variable de proceso flujo de entrada al sistema de flotación, durante el mismo periodo de tiempo en el cual fue muestreado el nivel operático de la celda. Es importante mencionar que para la fecha de muestreo de las tendencias del proceso aquí presentadas, la estrategia de control implementada y operativa en el sistema consiste en la regulación del nivel total de la celda de flotación con la válvula de control de caudal de admisión a la celda, con un simple lazo de control PID.

La regulación del caudal de salida de la celda está a cargo de la resistencia que ofrecen los filtros de cascarilla vegetal ubicados aguas abajo de la celda, regulados por presión con la válvula de control ubicada a la salida de cada uno de estos.

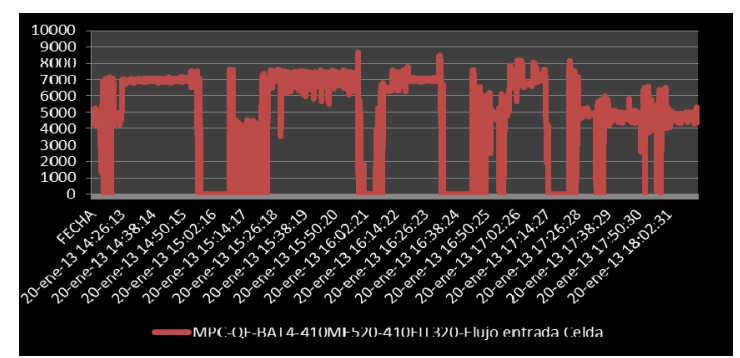

Fig. 3. Comportamiento perturbado de la variable de proceso flujo de entrada a la celda.

\section{EMULADO DEL SISTEMA FLOTACIÓN-FILTRACIÓN}

La construcción del sistema emulado, se ha obtenido a partir de la identificación dinámica de los sub-sistemas de manera individual, mediante ensayos escalón de tipo industrial, así como del levantamiento de información de ingeniería de los grupos de proceso tales como tanque de suministro a sistemas de flotación y sistema de flotaciónfiltración de agua.

El modelo desarrollado para el presente trabajo es de tipo caja gris, ya que cuenta con las características definidas para este tipo de modelo en tanto que parte de este se obtiene mediante leyes físicas e información de ingeniería constructiva, y otra parte, se ajusta mediante el empleo de ensayos industriales experimentales.

Adicionalmente, se han efectuado observaciones de las interacciones entre los sistemas de flotación y el sistema de suministro de fluido. De esta manera, se han identificado las perturbaciones que afectan el proceso, las cuales han sido incluidas en la construcción del modelo.
La técnica empleada para la determinación de la dinámica del comportamiento del nivel de fluido en la celda de flotación, se basa en la solución de la ecuación de balance volumétrico que ingresa y sale del tanque en virtud del tiempo. El balance de masa de un tanque de decantación de flujo continuo es:

$$
A \frac{d h(t)}{d t}=q_{\text {in }}(t)-q_{\text {out }}(t)
$$

La Ec. 1 describe como la variación del nivel de fluido de la celda con respecto del tiempo, a través de su área transversal, es igual al diferencial de caudal de entrada salida de la celda a través del tiempo.

Al integrar la Ec. 1, se obtiene la solución general para determinar el modelo integrante de la celda de flotación en su región operativa ( Ec. 2).

$$
h(t)=\frac{1}{A} \int_{0}^{T}\left(q_{\text {in }}(t)-q_{\text {out }}(t)\right) d t
$$

Con base en la Ec. 2, se construye el modelo matemático válido para la región operativa de desnate de crudo de la celda de flotación, la cual está comprendida en entre la altura de las tomas de proceso para el transmisor de nivel por presión diferencial ubicadas según la figura 4.

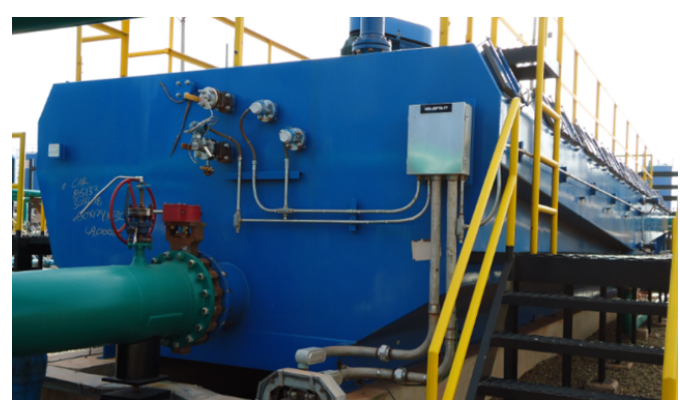

Fig. 4. Vista de perfil de la celda de flotación.

En la figura 5, se consigna una prueba step llevada a cabo para identificar la dinámica física del modelo de la celda de flotación. En la tendencia en rojo de la grafica, se aprecia la pendiente decreciente que describe el nivel de fluido de la celda, al desbalancear el caudal de entrada con respecto al de salida.

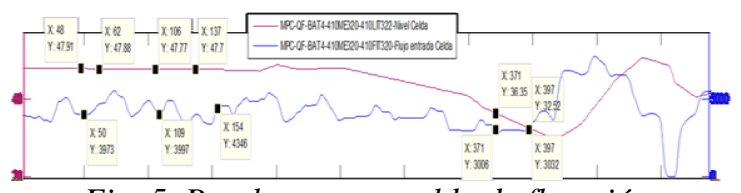

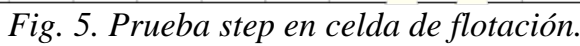


Con la identificación de la pendiente de descenso, en virtud del desbalance de caudal entrada salida, se obtiene la ganancia de integración $K_{p}$ del tanque, con la cual se puede describir la Ec.4, la cual puede ser empleada para emular el sistema en el dominio de la frecuencia.

$$
H(s)=K_{p} \frac{q_{\text {in }}(t)-q_{\text {out }}(t)}{s}[\%]
$$

Donde:
$K_{p} \quad$ Ganancia integrante de la celda
$H(s) \quad$ Nivel de fluido de la celda
$q_{\text {in }} \quad$ Caudal de entrada a la celda
$q_{\text {out }} \quad$ Caudal de salida de la celda

Para la construcción del sistema emulado sobre el software de programación de controladores de gama alta de Allen Bradley RS Logix, se ha tomado como base la Ec.3, en la que se han verificado los resultados de pruebas step efectuadas sobre este sistema, consignados en la figura 5.

Posterior a la obtención de la función de transferencia (FT) dinámica, adecuada para emular la celda en las unidades de ingeniería empleadas en el proceso real, se construye el modelo en bloques funcionales en el software RS Logix de Allen Bradley, como se visualiza en la figura 6 .

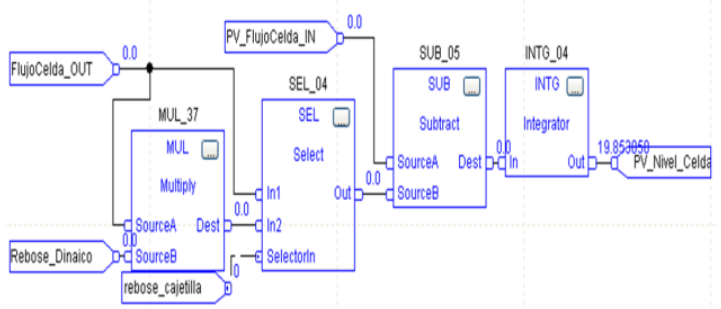

Fig. 6. Modelo dinámico de celda de flotación.

El filtro de lecho vegetal de la figura 7 es un recipiente hermético, el cual para la operación en los diferentes ciclos de trabajo, cuenta con una serie de tuberías y válvulas y así mismo tiene asociada una dinámica de presión de operación.

En la figura 8, se presentan los resultados de una de las pruebas step realizadas sobre el sistema de control de presión de los filtros de cascarilla vegetal, con lo cual se identifica la dinámica de cambio de presión del sistema, en su región de operación nominal.

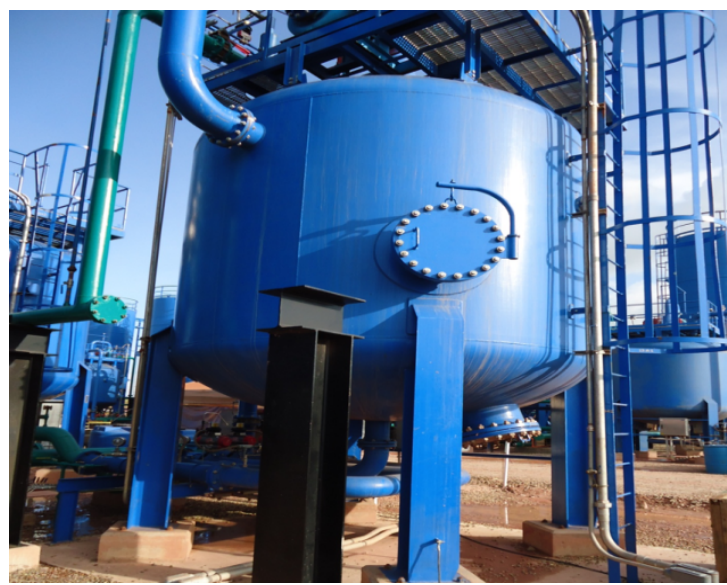

Fig. 7. Filtro de lecho vegetal.

Teniendo como referencia la figura 8 , se obtiene la función de transferencia del sistema mediante una aproximación a un sistema de primer orden más tiempo muerto.

$$
P(s)=\frac{K e^{-L s}}{\tau s+1}
$$

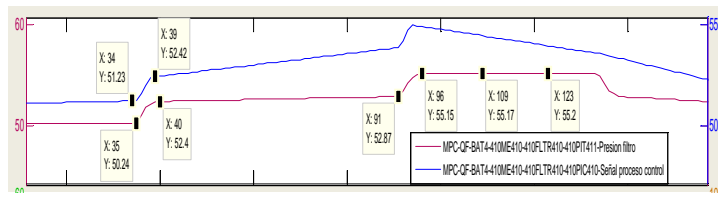

Fig. 8. Pruebas step en sistema de presión de filtros para agua clarificada.

Donde:

$P(s) \quad$ Presión del filtro.

$K \quad$ Ganancia estática del sistema.

$\tau \quad$ Constante de tiempo.

$L \quad$ Retardo de transporte del sistema.

$$
\tau=\frac{T_{s}}{4}
$$

De acuerdo con la identificación del lazo de presión, se determinó la función de transferencia de primer orden con bajo retardo de transporte tomado de la Ec. 4, expresada a continuación.

$$
C_{p}(s)=\frac{1.8 e^{-0.5 t}}{1.25 s+1}
$$

Se ha empleado además un modelo identificado de la transferencia de fluidos desde tanques Skim hacia sistemas de filtración y adicionalmente, se ha construido y emulado la curva presión caudal de bombas de transferencia de agua a filtros de lecho vegetal. 
El modelo construido se ha enlazado con la lógica de control asociada al sistema programado en el control distribuido y se ha presentado en interfaz de usuario HMI en software de la suite de Allen Bradley (figura 9).

\section{CONCEPTUALIZACIÓN DE LA ESTRATEGIA DE CONTROL AVANZADO}

La estrategia de control avanzado propuesta, es una alternativa para mantener los estándares de calidad en aguas a la salida de los sistemas de flotaciónfiltración y mantener la correcta operación del control de nivel y separación de aceite por desnate en facilidades de deshidratación de crudos.

La estrategia de control parte de la modificación del cuadro de control del sistema, pasando del control de nivel a la entrada de la celda con la válvula de control aguas arriba de la misma, a la regulación del suministro de fluido o caudal volumétrico a la entrada del sistema de flotación con la válvula proporcional a la entrada y adicionalmente, el control de nivel de la celda con las válvulas de control aguas abajo de los filtros de lecho vegetal, para garantizar el continuo desnate de aceite a través de las diferentes condiciones operacionales del sistema; como se presenta en la figura 9.

El establecimiento de la nueva estrategia no regulará de manera directa la presión de operación de los filtros, lo cual puede parecer preocupante en la medida en que estos podrían operar por fuera de su rango de operación de diseño.

Para brindar tranquilidad acerca de los valores de presión que asumirá el filtro al tener ligada su válvula de control a la regulación del nivel de la celda, se ha demostrado en el sistema emulado, la naturalidad con que la variable de proceso presión se acomoda a los valores de operación por diseño si se mantiene el caudal nominal operativo a través de cada uno de estos.

Para garantizar que el cuadro de control propuesto (figura 9) es viable en la aplicación práctica, se ha emulado el proceso en prototipo virtual desarrollado en la suite de software Allen Bradley, el cual reproduce de manera fiable el comportamiento dinámico del sistema de flotaciónfiltración y previamente se ha validado con la aplicación de la estrategia de control original de este.
El sistema de control propuesto, reduce el empleo de controladores PID, ya que en el sistema original se emplean tres controladores para regular de manera independiente la presión de cada filtro. En el sistema propuesto el uso de controladores PID se reduce a dos en total, eliminando el empleo de dos controladores, con lo cual se consigue bajar carga de procesamiento.

Aunque en aspecto se presenta más sencillo el control del sistema, en la práctica tiene inmersa la aplicación técnicas de control avanzadas que permiten el ajuste del sistema ante diferentes situaciones operativas del proceso, tales como la aplicación de supervisores difusos como mecanismo de adaptación de los controladores PID y la aplicación de ajustes en los balances volumétricos en operaciones de retro-lavado de filtros, basados en modelo hidráulico de transferencia de caudales individuales de los filtros de grasas y lodos.

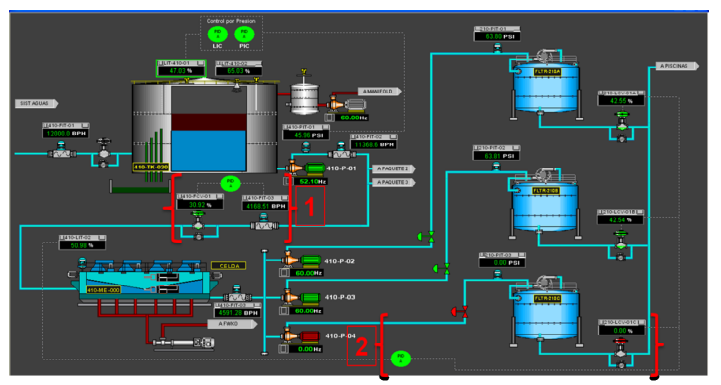

Fig. 9. Cuadro de control propuesto.

La regulación de caudal de entrada al sistema garantiza el desacople de disturbios producidos por otros sistemas de proceso aledaños. Ya que la dinámica del sistema es rápida debida al tamaño del tanque de flotación, y a los caudales de entradasalida del mismo, los cuales oscilan entre 2080 y 6250 [bls/h], un cambio de referencia o set point del flujo de entrada, obedece a la entrada o salida de un filtro de 2080 [bls/h] lo cual ocasiona una modulación de la válvula de control de flujo a la entrada de más o menos un de un cuarto de la apertura total ante el cambio de referencia.

\section{DESARROLLO DE LA ESTRATEGIA DE CONTROL AVANZADO}

La condición dinámica o de velocidad alta de las variables del proceso, requiere acciones de control oportunas y sincronizadas de los controladores de flujo de admisión de la celda y nivel de fluido de la misma. 
Para alcanzar este nivel de sincronismo, ante cualquier circunstancia operativa del sistema se implementó sobre los controladores de nivel y flujo supervisores difusos basados en el conocimiento experto de los autores, los cuales tienen por funcionalidad el ajuste de la sintonía del lazo permitiendo adaptabilidad y manteniendo estable el tanque de flotación para asegurar la remoción de aceite en los skimers.

El supervisor difuso se ajusta la acción de control a los diferentes escenarios operativos del sistema de flotación-filtración. Así por ejemplo si el sistema se encuentra operando en condiciones estables y no perturbadas, el requerimiento de la acción de control es suave y lento para brindarle al sistema las condiciones mas favorables para la remoción de aceites. Bajo condiciones en las cuales hay tendencia de desviación de las variables de proceso flujo y nivel de sus valores de consigna, se requiere de una acción de control encaminada a retomar el curso de estas variables para mantener la estabilidad del proceso.

En la figura 10, se presenta el esquema general del supervisor difuso como mecanismo de adaptación de controladores PID, el cual tiene como variable de entrada el error de la variable de proceso y, a través de este, efectúa su mecanismo de ajuste de constantes PID.

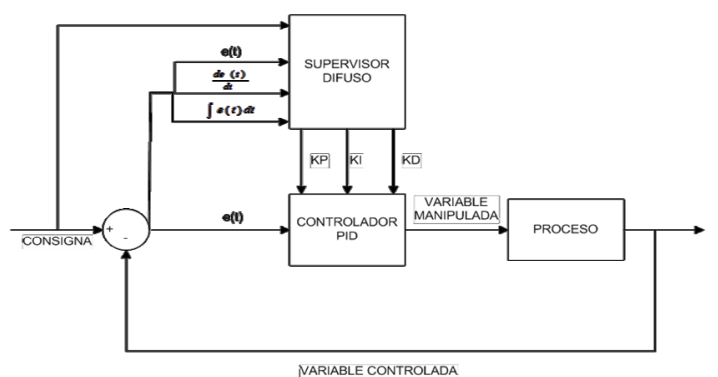

Fig. 10. Supervisor difuso de controladores PID.

Con la variable lingüística de fuzzificación del error (figura 11), se pretende cuantificar que tan grande es el error para el sistema, para indicarle al mecanismo difuso cual debe ser la acción de control que encamine a la variable de proceso a su valor de estado estable de manera suave y a su vez oportuna

Una vez cuantificado el grado de pertenencia de la variable linguiística de entrada, se hace necesario someter esta cuantificación o grado de pertenencia del conjunto difuso a la base de reglas, la cual determinará la necesidad del proceso de ejecutar la acción de control apropiada para cada condición operativa. De acuerdo con la desviación de la variable de proceso, el mecanismo de ajuste decide sobre la conveniencia de asignar agresividad al controlador o, por el contrario suavizar la acción de control.

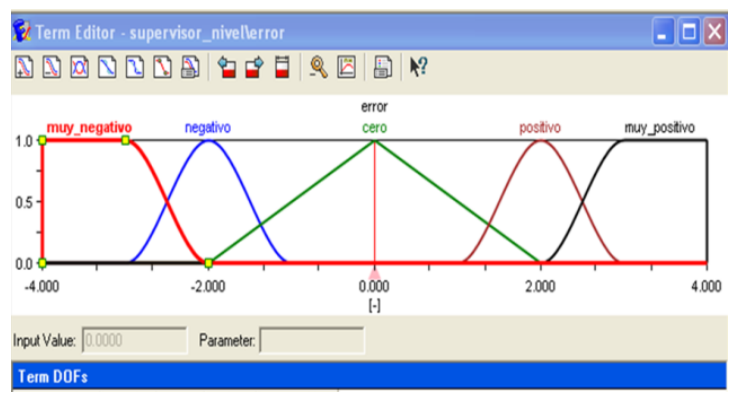

Fig. 11. Mecanismo de fuzzificación.

Adicionalmente a la necesidad de ajuste o adaptación del controlador, es de gran importancia la tabulación de ajustes de sintonía del experto, quien previamente ha efectuado pruebas on-line sobre la planta para verificar los mejores puntos operativos de los controladores de acuerdo con la región de control por la cual se atraviese en un tiempo determinado.

Para el ajuste de constantes PID, se ha empleado el método lambda que es muy conocido en el ámbito industrial. Este método de ajuste de controladores PID garantiza que las variables de proceso encuentren su punto de consigna con el mínimo de fluctuación y sobrepaso en referencia al valor de consigna o set point.

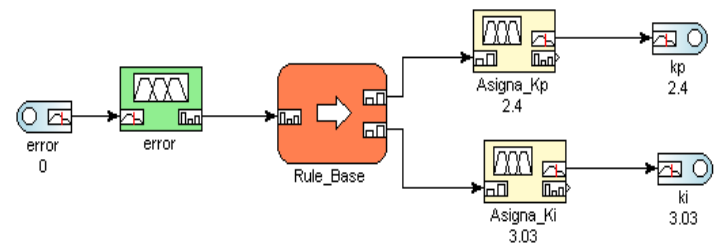

Fig. 12. Estructura Fuzzy Designer supervisor PI.

En la figura 12, se muestra la estructura del mecanismo de supervisión difuso para ajuste de controladores PID, el cual fue desarrollado en la en herramienta Fuzzy Designer de Allen Bradley.

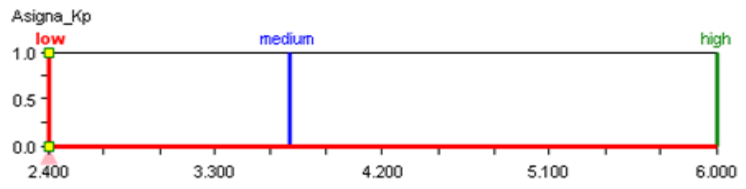

Fig. 13. Defuzzificación de la variable lingüística. 
De acuerdo como lo ilustra la figura 13, posterior a la base de reglas programada para el supervisor adaptativo difuso, se conecta el componente de defuzzificación de la variable lingüística, el cual, para el caso especifico de la estrategia de control adaptativo es empleada para el control del nivel del sistema de flotación-filtración

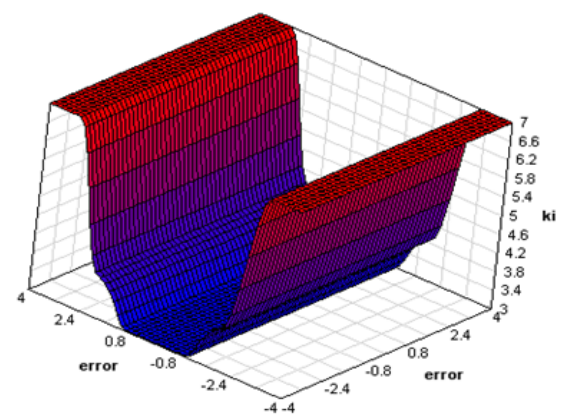

Fig. 14. Superficie de adaptación de ganancias.

\section{RESULTADOS}

De manera general, la estrategia de control conceptualizada y desarrollada ha disminuido de manera notaria la oscilación de las variables de proceso en el sistema real, tal como se formuló a nivel de emulación. En el caso particular de este desarrollo investigativo, se han efectuado pruebas en operación normal del sistema, las cuales se han comparado con los resultados del sistema emulado para verificar la fiabilidad de este y la potencialidad de la estrategia de control.

En la figura 15, se presentan las tendencias de flujo de entrada al proceso y nivel de la celda de flotación, ante las condiciones de operación específicas del sistema.

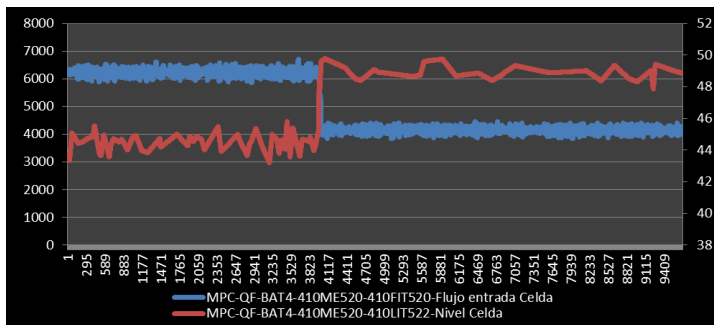

Fig. 15. Data muestral de flujo y nivel del sistema de flotación real.

En la figura 16, se aprecia como la variable de proceso flujo tiende a seguir una distribución normal con media en el setpoint nominal de operación para las dos condiciones operativas estudiadas, las cuales corresponden a la operación con tres filtros en un periodo de tiempo y posteriormente con dos filtros activos.

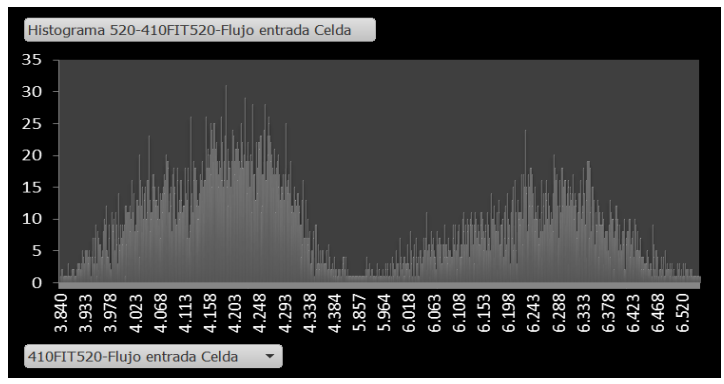

Fig. 16. Distribución de la variable de proceso flujo con estrategia de control implementada.

En el histograma mostrado, se aprecian las frecuencias de repetición de valores de la muestra representativa consignada en la figura 15, en la cual la media muestral para la operación con tres filtros es de 6254 barriles por hora y la media muestral para la operación con dos filtros es de 4169 barriles por hora. Los resultados de la media dejan en evidencia la precisión con que el sistema de control mantiene la variable de proceso sobre sus setpoints nominales.

\subsection{Resultados ante cambios de referencia de $66 \%$ a $100 \%$ de capacidad nominal}

En la figura 17, se presenta en detalle el comportamiento real del evento de cambio de la referencia del caudal de entrada de la celda de flotación y el nivel total de fluido acomodándose para asegurar la operación de desnate para el nuevo valor de caudal ingresado al tanque de flotación, ante la acción de puesta en servicio de un filtro del lecho vegetal.

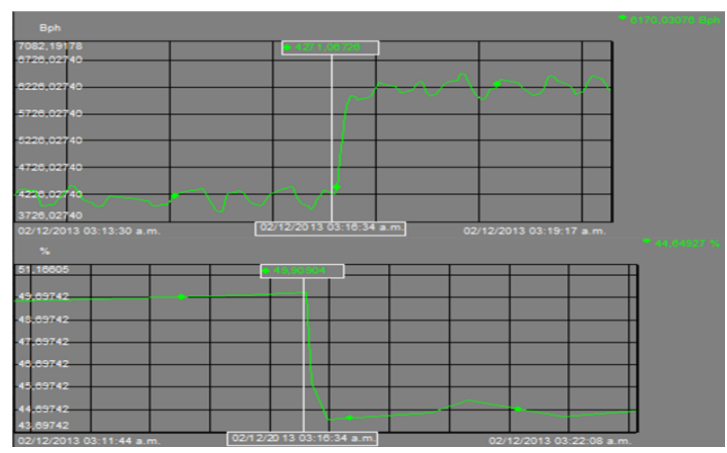

Fig. 17. Respuesta dinámica de la variables flujo y nivel del sistema real ante cambio de referencia.

Así mismo, en la figura 17, se efectúa la sobre posición de tendencias obtenidas en proceso real y generadas por el sistema emulado, las cuales de manera visual muestran el comportamiento adecuado de los controladores y el comportamiento acertado del sistema emulado. 


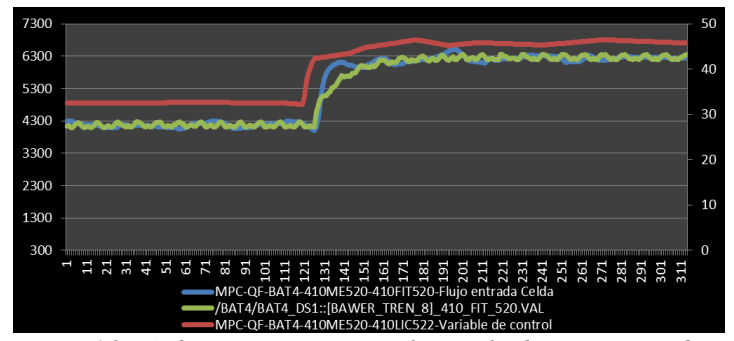

Fig. 18. Sobre-posición real-emulado ante cambio de referencia del flujo de admisión.

\section{CONCLUSIONES}

En el presente trabajo, se generaron modelos de tipo caja gris que reprodujeron las condiciones dinámicas presentes en la operación de sistemas de separación de grasas y aceites por flotación. Se consiguió modelar y emular un sistema de flotación-filtración típico de la industria petrolera y se validaron los modelos matemáticos obtenidos por técnicas científico-experimentales.

Los modelos matemáticos que permitieron el desarrollo del sistema de flotación emulado en plataformas de software industrial, son la base de una poderosa herramienta para el estudio dinámico de los sistemas típicos de separación de crudos por flotación presentes en plantas de deshidratación propias de la industria petrolera. Con el presente desarrollo se dota a la industria petrolera con un paquete de investigación de condiciones dinámicas de proceso el cual puede ser empleado para la refinación de técnicas de control que permitan maximizar la eficiencia de los procesos.

La presente investigación desarrolló una nueva filosofía de control para sistemas de Celdas de Flotación y tanques Skim fundamentado en la regulación del suministro de fluidos y el establecimiento de controladores adaptativos apoyados de técnicas de diseño difuso encaminadas a la acomodación de las ganancias de controladores PID convencionales presentes en la mayoría de las facilidades productoras de los campos petroleros.

Se conceptualizaron y construyeron instrucciones programadas las cuales basadas en el modelo dinámico de las bombas de transferencia de fluidos hacia tanques de filtración sellados, adicionaron bondades como el balance volumétrico de caudales de entrada salida al sistema de flotación, bajo condiciones de retro-lavados del lecho filtrante cuando se requiere bajar la concentración de partículas de grasa en los filtros ya sea por saturación del lecho o simplemente por cumplimiento de los tiempos estipulados por la operación para el lavado de este.

El controlador PID adaptativo con supervisor difuso entrega características de respuesta mejoradas comparado con un controlador PID convencional, dichas características están fundamentadas en la velocidad de respuesta del lazo de control ya que el supervisor adapta la acción PID a la manera como se genera el error de la variable de proceso, en virtud de la condición de no linealidad del sistema.

\section{RECONOCIMIENTO}

Los autores agradecen el apoyo de Pacific Rubiales Energy, durante el desarrollo del presente trabajo investigativo.

\section{REFERENCIAS}

P. Kämpjrvi, S.-L. - Jämsä Jounela. 2003. Level control strategies for flotation cells.Minerals Engineering.

B. J. Shean, J.J. Cilliers. 2011. A review of froth flotation control. Mineral Processing

Carvalho, M.T., Durão, F., 2002. Control of a flotation column using fuzzy logic inference. Fuzzy Sets and Systems.

Núñez F. 2010. Hierarchical hybrid fuzzy strategy for column flotation control. Minerals Engineering.

Jocelyn B. 2009. Column flotation simulation and control: An overview. Elsevier, Minerals Engineering.

Jämsä-Jounela, S.-L., Dietrich, M., Halmevaara, K., Tiili, O., 2003. Control of pulp levels in flotation cells. Control Engineering Practise.

Pardo García, A; Díaz Rodríguez, J. L. 2004 Fundamentos en sistemas de control automático. Universidad de Pamplona.

Astrom Karl J. Advanced PID Control. ISA Library of congress. United States of America 2005

Richerand, F. An historical perspective on produced water treatment. Society of Petroleum Engineering (SPE) 2012.

Wade H. Basic and Advanced Regulatory Control:System Design and Application. 2a Edition. ISA 2004

Yinfei L. Simulating a fuzzy level controller for flotation columns. Mining Science and Technology (China), Elsevier, 2011. 